\title{
Corela
}

Cognition, représentation, langage

HS-16 | 2015

Diversité des pratiques de recherche en science du langage

\section{L'objet-langue et l'appropriation dans un cadre institutionnel : une approche « raisonnée » des acquisitions syntaxiques en allemand L2}

\section{Catherine Felce}

\section{OpenEdition}

\section{Journals}

Édition électronique

URL : http://journals.openedition.org/corela/3895

DOI : $10.4000 /$ corela.3895

ISSN : $1638-573 \mathrm{X}$

Éditeur

Cercle linguistique du Centre et de l'Ouest - CerLICO

Référence électronique

Catherine Felce, «L'objet-langue et l'appropriation dans un cadre institutionnel : une approche « raisonnée » des acquisitions syntaxiques en allemand L2 », Corela [En ligne], HS-16 | 2015, mis en ligne le 30 juin 2015, consulté le 02 mai 2019. URL : http://journals.openedition.org/corela/3895 DOI : 10.4000/corela.3895

Ce document a été généré automatiquement le 2 mai 2019.

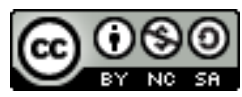

Corela - cognition, représentation, langage est mis à disposition selon les termes de la licence Creative Commons Attribution - Pas d'Utilisation Commerciale - Partage dans les Mêmes Conditions 4.0 International. 


\title{
L'objet-langue et l'appropriation dans un cadre institutionnel : une approche " raisonnée " des acquisitions syntaxiques en allemand L2
}

\author{
Catherine Felce
}

\section{Introduction}

1 Le travail de recherche en didactique des langues que nous menons vise à élaborer des pratiques susceptibles de favoriser chez des apprenants peu avancés de l'allemand L2 la mise en place d'une structure syntaxique particulière, caractérisée par la place seconde $d u$ verbe fléchi et l'ouverture de l'énoncé par un constituant autre que le sujet. Notre questionnement s'est ainsi construit autour des pôles que constituent la langue, son appropriation par l'apprenant et les modalités de l'intervention enseignante. Sur le plan de la langue, une analyse purement phrastique et descriptive ne permet pas de saisir ce qui préside au choix de l'élément en ouverture de l'énoncé et il est pour cela nécessaire de recourir à des catégories textuelles et aux critères pragmatiques qui interviennent dans l'organisation informationnelle des constituants. En ce qui concerne les processus d'appropriation, les résultats issus de la Recherche en Acquisition des Langues (RAL) et les modèles psycholinguistiques sur lesquels elle s'appuie permettent d'appréhender les aspects intra-psychiques qui sous-tendent la production langagière en L2 et la construction de l'interlangue des apprenants. Notre contribution vise à mettre en lumière la manière dont un faisceau de théories contribuent à informer la didactique, à construire un objet de recherche, et à élaborer une intervention de terrain « raisonnée $»^{1}$. 


\section{Une approche linguistique plurielle}

2 Il est désormais un fait acquis de considérer la langue non plus comme un simple registre de formes soumises à des règles dont il s'agit de maîtriser la complexité croissante mais bien comme un répertoire de ressources, certes normées, mais également dépendantes de situations d'usage et par conséquent ancrées dans des contextes et dans des discours (Narcy-Combes, 2005 : 58). Dans la construction de l'énoncé en allemand, Poitou (2010) distingue différents niveaux de structuration dont l'interaction permet la linéarisation d'un énoncé formulé : une structure syntagmatique qui reflète les relations entre les signifiés, une structure topologique de surface, et une organisation informationnelle. Si l'on considère que l'enseignement cherche à promouvoir chez l'apprenant non pas la simple production de phrases isolées et correctes mais bien des discours-textes pertinents, il apparait nécessaire d'avoir également recours à des notions qui ne se cantonnent pas à la grammaire de la phrase mais rendent justice à la réalité du fonctionnement de la langue. Nous nous plaçons dans une perspective fonctionnelle de la grammaire telle qu'elle est mise en avant dans la Grammatik der deutschen Sprache de Zifonun et al. (1997: 2) et qui met des phénomènes syntaxiques en relation avec leur fonctionnement pragmatico-sémantique.

\subsection{Structure topologique : hétérogénéité dans l'occupation de la position préverbale}

3 Pour décrire l'organisation des constituants de l'énoncé en allemand, on a généralement recours à la notion de "champs» et à un découpage qui correspond à un modèle topologique adopté dans la plupart des descriptions grammaticales (Dürscheid, 2010 : 88-94). Ce découpage est illustré dans le tableau ci-dessous et deux caractéristiques importantes apparaissent :

4 - Le caractère contraignant du placement verbal: la forme verbale fléchie occupe nécessairement la deuxième position dans l'énoncé assertif et l'élément non-fléchi en position finale referme « la pince verbale » qui enserre les constituants du Mittelfeld.

5 - L'hétérogénéité des éléments en ouverture, c'est-à-dire dans le Vorfeld. Les éléments en position préverbale témoignent en effet d'une grande variabilité. Le Vorfeld n'est pas une position syntaxiquement déterminée (Zifonun et al., 1997 : 1584) et réservé au sujet mais peut être occupé par différents constituants plus ou moins complexes et dans ce cas, le sujet se retrouve positionné à l'intérieur de la " pince verbale ", après le verbe fléchi, ce qui explique que les chercheurs acquisitionnistes parlent généralement d' «inversion »² pour désigner cette construction syntaxique particulière. En ce qui concerne le Vorfeld, des facteurs pragmatiques régissent son occupation et Dürscheid (2010:98) souligne à ce propos que la fonction pragmatique n'est pas strictement déterminée, il s'agit d'une donnée relative, dépendante du contexte situationnel ou textuel dans lequel s'inscrit l'énoncé. 


\begin{tabular}{|c|c|c|c|c|c|}
\hline $\begin{array}{l}\text { Vor-vorfeld } \\
\text { [champ } \\
\text { externe } \\
\text { gauche] }{ }^{3}\end{array}$ & $\begin{array}{l}\text { Vorfeld } \\
\text { [champ } \\
\text { initial] }\end{array}$ & $\begin{array}{l}\text { rechte } \\
\text { Satzklammer } \\
\text { [forme verbale } \\
\text { finie] }\end{array}$ & $\begin{array}{l}\text { Mittelfeld } \\
\text { [champ } \\
\text { médian] }\end{array}$ & $\begin{array}{l}\text { linke } \\
\text { Stazklammer } \\
\text { [forme verbale } \\
\text { non finie] }\end{array}$ & $\begin{array}{l}\text { Nachfeld } \\
\text { [champ } \\
\text { terminal] }\end{array}$ \\
\hline & $\begin{array}{l}\text { Position } \\
\text { préverbale }\end{array}$ & $\begin{array}{l}\text { Satzklammer } \\
\text { [sentence } \\
\text { bracket] }\end{array}$ & & & \\
\hline & $\mathrm{x}$ & V2 & & V END & \\
\hline \multirow[t]{3}{*}{$\begin{array}{l}\mathrm{Ja}, / \text { und } \\
\text { Yes, /and }\end{array}$} & $\begin{array}{l}\text { (1)ich } \\
\text { I }\end{array}$ & $\begin{array}{l}\text { fahre } \\
\text { go }\end{array}$ & $\begin{array}{l}\text { mit Peter } \\
\text { nach } \\
\text { Hamburg } \\
\text { with Peter to } \\
\text { Hamburg }\end{array}$ & & \\
\hline & $\begin{array}{l}\text { (2) Ich } \\
\text { I }\end{array}$ & $\begin{array}{l}\text { bin } \\
\text { am }\end{array}$ & $\begin{array}{l}\text { gestern mit } \\
\text { Peter nach } \\
\text { Hamburg } \\
\text { yesterday } \\
\text { with Peter to } \\
\text { Hamburg }\end{array}$ & $\begin{array}{l}\text { gefahren } \\
\text { gone }\end{array}$ & $\begin{array}{l}\text {, weil } \\
\text {, because }\end{array}$ \\
\hline & (3) Gestern & bin & $\begin{array}{l}\text { ich nach } \\
\text { Hamburg }\end{array}$ & gefahren & - \\
\hline & (4)Mit Peter & bin & $\begin{array}{l}\text { ich nach } \\
\text { Hamburg }\end{array}$ & gefahren & - \\
\hline
\end{tabular}

Tableau 1 : Un modèle topologique de l'énoncé assertif

\subsection{Perspective communicative et hiérarchisation informationnelle}

6 La syntaxe positionnelle ne reflète donc pas en allemand une hiérarchie des catégories grammaticales mais une progression informationnelle qui va du connu vers le nouveau. Le choix variable de l'élément préverbal participe de la mise en relief de certaines unités et témoigne de l'ancrage situationnel de l'énoncé produit.

$7 \quad$ Le code laisse au locuteur une grande marge de liberté pour linéariser les divers éléments de son énoncé (...) la liberté laissée par le code est surtout exploitée par ce que nous avons appelé la fonction de perspective : la distribution des diverses unités de l'énoncé est liée à l'importance relative qu'elles ont dans l'information transmise. Cette importance ou "dynamisme» dépend des données du contexte et de la situation d'une part et des intentions communicatives du locuteur d'autre part. (Schanen \& Confais, 1989 : 583-584)

Les options de linéarisation peuvent correspondre à une suite " neutre » qui reprend la progression informationnelle classique: thème d'abord (ce dont il est question) puis rhème (information apportée sur ce dont il est question), mais le locuteur germanophone 
peut aussi faire usage des possibilités d'une linéarisation plus ou moins " marquée » au gré de ses intentions communicatives et de la hiérarchisation qu'il établit entre des unités informatives.

Il s'agit de l'intention du locuteur d'établir par le biais de moyens grammaticaux des points forts dans son énoncé, de différencier les degrés d'importance des mots et ainsi de hiérarchiser les valeurs informatives des éléments de la phrase ${ }^{5}$. (Blumenthal, 1997: 31)

(1) Morgen fahre ich nach Hamburg. [demain je vais à Hambourg]

(2) Nach Hamburg fahre ich morgen. [je vais demain à Hambourg]

En (1) l'unité informative la plus importante est la destination nach Hamburg [à Hambourg] alors qu'elle porte sur l'indication temporelle morgen [demain] en (2). Schanen et Confais (1989 : 584) ramènent ces deux énoncés à deux questions distinctes qui peuvent être formulées de façon plus ou moins implicite. Pour (1) la question sousjacente porterait sur la destination :

(1) Wohin fährst du morgen ? [où vas-tu demain ?] Morgen fahre ich 'nach Hamburg alors que pour (2) il s'agirait de connaître une destination :

(2) Wann fährst du nach Hamburg? [quand vas-tu à Hambourg ?] Nach Hamburg fahre ich ${ }^{\circ}$ morgen.

On le voit à travers ces exemples, si l'énoncé (1) - qui affiche une linéarisation plus neutre - peut être formulé dans des contextes variés, la recevabilité de l'énoncé (2) dépend quant à elle du contexte particulier que constitue l'état de savoir de l'interlocuteur. Les intentions communicatives sont encore différemment marquées dans les cas de dislocation, c'est-à-dire quand il y a déplacement vers l'avant d'un élément d'un groupe qui permet de retarder l'apparition d'un autre constituant de ce même groupe (souvent une négation ou un quantificateur) qui garde quant à lui sa place structurale et, ce faisant, se voit accorder une valeur informative plus grande en restant en fin d'énoncé.

(3) $\mathrm{Pizza}^{\circ}$ bezahle ich nur ${ }^{\circ}$ meine [des pizzas, je ne paye que la mienne]

(4) Eine ${ }^{\circ}$ Schwimmweste trug sie ${ }^{\circ}$ nicht $^{6}$ [ un gilet de sauvetage, elle n'en portait pas]

13 On le voit à travers les équivalents donnés en français, un élément informatif est mis en exergue par son déplacement vers la première position, non pas pour être mis en valeur mais pour provoquer un effet de retardement par rapport à l'information attendue : celle que je paye (des pizzas), c'est la mienne (meine), quant au gilet de sauvetage, c'est son absence qui est ainsi particulièrement soulignée (nicht). La liberté de choix de l'élément placé en ouverture permet ainsi une adaptation de la transmission informationnelle aux besoins de l'interlocuteur et une hiérarchisation des unités informatives conforme aux intentions communicatives du locuteur.

\subsection{Principes textuels de cohérence et de cohésion}

Martine Dalmas (2005 : 101) relativise cependant la liberté de choix dont disposerait le locuteur pour la restreindre à juste titre à la liberté que permet le texte lui-même et Gunhild Samson rappelle que des choix de linéarisation soumis à des critères communicationnels participent également de l'organisation textuelle / discursive.

Sous l'angle de la production de texte, cet aspect pragmatique, lié aux effets de l'interaction entre les partenaires sur le discours / texte, se répercute sur la dynamique textuelle. D'un côté, les éléments en tête de l'énoncé qui traduisent, par un enchaînement 
soit neutre, soit marqué, la visée communicative du locuteur, produisent également, par leur contenu lexical, l'enchaînement textuel et instaurent la cohérence sémantique du texte. (Samson, 2004 :187)

À ce titre, le choix des éléments qui assurent l'ouverture des énoncés est lié à la progression thématique et à la cohérence du discours produit, ainsi qu'à la cohésion entre des énoncés qui ne peuvent être considérés comme isolés. Dalmas nous invite à regarder au-delà des limites de l'énoncé ${ }^{7}$ afin de comprendre le fonctionnement des éléments placés dans le Vorfeld. Si on considère les énoncés produits non plus de façon isolée mais dans leur succession, il apparaît que l'élément occupant la position préverbale assure bien souvent un lien entre les énoncés par un phénomène de reprise plus ou moins explicite.

[...] alors que celle-ci (l'occupation de la première position dans la phrase) est régie, dans les langues qui privilégient le sujet, essentiellement par des critères morphosyntaxiques (accord verbe-sujet), l'occupation de la première position au sein même de la phrase se réalise en allemand selon des critères textuels: l'ancienne information (le topic) sert de raccord avec le pré-texte et est placé en début de phrase ${ }^{8}$. (Schmidt 1996 : 114 cité dans : Diehl et al., $2000: 67$ )

17 Zifonun et al. précisent enfin que l'occupation du Vorfeld joue un rôle important pour assurer la continuité thématique et que cette dernière ne s'instaure pas en allemand à partir d'une catégorie syntaxique donnée. Si le sujet fait certes partie des données thématiques, il est important de souligner à nouveau qu'il n'est pas pour autant une entité destinée par nature à occuper la position préverbale.

En marquant le début d'une unité thème-rhème dans un mode déclaratif le Vorfeld est d'une

certaine façon à même de réaliser la jonction avec ce qui précède, c'est-à-dire qu'il constitue la place privilégiée pour des constituants qui permettent la poursuite ou le développement d'un thème. (...) La continuité thématique n'est pas liée à des unités syntaxiques précises $^{9}$. (Zifonun et al., $1997:$ 1642)

\subsection{Positionnement linguistique entre norme et usage}

20 C'est par conséquent un double fonctionnement qui intervient dans la construction de l'énoncé assertif en allemand: d'une part, des contraintes positionnelles affectent le placement verbal et contraignent la correction grammaticale des énoncés, d'autres impératifs moins strictement normés conditionnent également la construction plus globale du discours selon les principes textuels de cohérence et de cohésion, et d'autre part enfin, l'exploitation des libertés à l'ouverture permet une modulation informationnelle qui doit selon nous faire l'objet d'un enseignement dans la mesure où elle est spécifique à la L2. En effet, les présentations grammaticales des manuels scolaires français font une règle d'or du placement verbal et négligent de se pencher sur les facteurs qui déterminent des choix plus liés à un usage contextualisé qu'à des règles normatives. Pour le chercheur comme pour l'enseignant la compréhension du fonctionnement de la langue objet d'apprentissage nécessite de faire la synthèse ${ }^{10}$ entre la description du système de la LC et une vision plus fonctionnaliste capable de rendre compte de son emploi sous la forme d'une production de discours/textes ${ }^{11}$. 


\section{Apports de la RAL}

21 La question de l'acquisition de l'ordre des mots a été largement traitée par les chercheurs en RAL et a fait l'objet d'études de grande envergure (Etude Zweitspracherwerb Italienischer und Spanischer Arbeiter - ZISA - Meisel, Clahsen \& Pienemann, 1981) dont les résultats ont ensuite été mis en relation avec des questions relatives à l'enseignement des langues. Au-delà des seuls résultats obtenus, la RAL a également contribué à faire des théories et des modèles psycholinguistiques des outils indispensables à la compréhension des processus de production langagière et d'apprentissage de L2. Elle peut être considérée comme science de fondement pour la didactique des langues, indissociable de la réflexion théorique qui permet la construction de l'objet de recherche.

\subsection{Des résultats concernant des itinéraires d'appropriation}

Une des principales contributions des études acquisitionnelles menées dans les années 80 auprès d'adultes migrants a été de mettre en évidence une échelle implicationnelle des stades d'acquisition relatifs à l'acquisition de l'ordre des mots. Cette échelle s'est également avérée valide dans les travaux ultérieurs de Pienemann effectués en contexte guidé et dans une étude de grande envergure menée auprès d'apprenants francophones de l'allemand dans les écoles genevoises (Deutsch in Genfer Schulen DiGS - Diehl et al., 2000). Pienemann propose une interprétation psycholinguistique à cette succession de stades que l'enseignement ne peut infléchir. Selon lui, il s'agit pour les apprenants d'être en mesure de traiter (to process) des formes langagières et par conséquent d'avoir acquis les procédures qui rendent possible ce traitement - procédures qui ne se développent qu'à condition que les pré-requis antérieurs soient en place.

\begin{tabular}{|l|l|}
\hline $\begin{array}{l}\text { Etude ZISA } \\
\text { (Pienemann, Clahsen, Meisel }\end{array}$ & $\begin{array}{l}\text { Etude DiGS } \\
\text { (Diehl et al., 2000) }\end{array}$ \\
\hline $\begin{array}{l}\text { 1-Stage } \mathrm{x}=\text { Canonical Order } \\
\text { die kinder spielen mim ball } \\
\text { [the children play with the } \\
\text { ball] }\end{array}$ & I Phrase principale (sujet - verbe) \\
\hline $\begin{array}{l}\text { 2- Stage } \mathrm{x}+1=\text { Adverb } \\
\text { Preposing (ADV) } \\
\text { da kinder spielen } \\
\text { [there children play] }\end{array}$ & $\begin{array}{l}\text { II Principales coordonnées } \\
\text { Questions partielles } \\
\text { Questions globales }\end{array}$ \\
\hline $\begin{array}{l}\text { 3- Stage } \mathrm{x}+2=\text { Verb } \\
\text { Separation (SEP) } \\
\text { alle kinder muss die pause } \\
\text { machen } \\
\text { [all children must the break } \\
\text { have] }\end{array}$ & $\begin{array}{l}\text { III Séparation des composantes fléchies et non- fléchies de la } \\
\text { forme verbale (Verbalklammer) }\end{array}$ \\
\hline
\end{tabular}




\begin{tabular}{|c|c|}
\hline $\begin{array}{l}\text { 4- Stage } \mathrm{x}+3=\text { INVERSION } \\
\text { (INV) } \\
\text { dann hat sie wieder die } \\
\text { knoch gebringt } \\
\text { [then has she again the bone } \\
\text { bringed] }\end{array}$ & IV Subordonnée \\
\hline $\begin{array}{l}\text { 5- Stage } \mathrm{x}+4=\text { Verb Final (V- } \\
\text { END) } \\
\text { er sagt, dass er nach hause } \\
\text { kommt } \\
\text { [he says that he home comes] }\end{array}$ & $\begin{array}{l}\text { V Inversion (X-verbe-sujet) } \\
\text { Structure propositionnelle de I à V acquise }\end{array}$ \\
\hline
\end{tabular}

Tableau 2 : Séquences acquisitionnelles en milieu naturel et en contexte institutionnel

23 À travers la théorie qu'élabore Pienemann (Processability Theory) (Pienemann, 1999) l'acquisition se présente comme une construction graduelle dans laquelle l'accession à une étape supérieure est soumise à l'automatisation des procédures mises en place au stade précédent.

L'acquisition d'une langue inclut l'acquisition des savoir-faire procéduraux nécessaires au traitement de celle-ci. Il s'ensuit que la séquence de développement de la LC chez l'apprenant est déterminée par la séquence de développement des processus de traitement nécessaires à l'utilisation des composantes de la LC ${ }^{12}$. (Pienemann $2005: 2$ )

Dans le cas de l'inversion, les apprenants de l'allemand L1 partent d'une structure de base de type SOV qui rend directement accessible la procédure permettant l'inversion; en revanche, la typologie du français comme langue source impose aux apprenants de l'allemand L2 un plus grand nombre d'étapes intermédiaires afin de se détacher progressivement de l'ordre SVO.

\subsection{Les aspects intra-psychiques de l'appropriation}

La robustesse de la succession des stades montre que le travail cognitif fourni par les apprenants ne se plie pas nécessairement au rythme d'apprentissage dicté par la progression proposée par les manuels. En favorisant l'incursion des paramètres intrapsychiques dans les critères à prendre en compte dans l'apprentissage, les résultats des travaux issus de recherches acquisitionnistes ouvrent la voie à une possible synergie entre les activités d'apprentissage et le travail cognitif des apprenants de L2 telle que Pierre Bange (2005) l'appelle de ses vœux.

Certains travaux en RAL rejoignent clairement des préoccupations pédagogiques et contribuent à la réflexion sur les modalités de l'intervention enseignante afin que celle-ci puisse influencer les processus acquisitionnels ou le traitement de l'input en rendant saillants des phénomènes que l'apprenant ne percevrait sinon pas. L'intégration d'un travail axé sur la forme (Focus on Form) ou d'activités de conscientisation au sein de tâches communicatives fait partie de cette manipulation destinée à faciliter le repérage (noticing) par un guidage attentionnel ou à automatiser certaines procédures par le biais d'une pratique contrôlée de manière explicite (Dörnyei, 2009 : 280-302). 
28 La question de l'interface entre connaissances implicites et explicites est cruciale en RAL et l'est aussi pour les spécialistes de l'enseignement / apprentissage des langues qui cherchent à déterminer le rôle des d'un travail formel ou métalinguistique dans la construction de savoir-faire procéduraux. Si dans le cas de la L1, un apprentissage implicite permet d'assurer l'acquisition d'une compétence native, ceci s'avère insuffisant quand il s'agit de la L2. Les connaissances explicites et une attention prêtée à la forme constituent un ensemble de ressources additionnelles qui permettent à l'apprenant de se détacher des procédures acquises pour le traitement de sa L1 (Ellis, 2011:45). Il fait aujourd'hui consensus que l'apprentissage de la L2 ne saurait faire l'économie d'une composante explicite (Dörnyei, 2009 : 175) et qu'il ne s'agit plus d'opposer ce qui relève de l'implicite et de l'explicite dans l'apprentissage et la production langagière en L2 mais plutôt d'optimiser leur coopération. L'enjeu pour les enseignants étant de réussir à trouver un équilibre entre les buts communicationnels et les objectifs d'apprentissage, c'est-à-dire de proposer des activités formelles ou métalinguistiques qui ne compromettent pas les interactions et ne prennent pas le dessus sur l'action langagière (Skehan \& Foster, 2001).

\subsection{Des modèles et des outils permettant d'appréhender la production langagière et d'orienter l'apprentissage de L2}

Pour décrire les processus cognitifs à l'œuvre dans la production langagière, il est courant de se reporter au modèle élaboré par Levelt (1989) dans lequel on distingue trois instances, correspondant chacune à trois étapes clés de l'élaboration d'un message verbal. Une phase de conceptualisation consiste à préparer un message préverbal destiné dans une seconde étape à être encodé au niveau lexical et grammatical avant d'être finalement articulé. La production langagière ne se limite donc pas à un processus de mise en mots, certes il peut manquer à l'apprenant des ressources lexicales spécifiques à la L2 et nécessaires à l'encodage mais la conceptualisation des événements relève également de principes spécifiques à la langue (von Stutterheim et al., 2002) et sur lesquels il semble utile de travailler avec les apprenants.

D'autres chercheurs ont analysé des performances d'apprenants selon trois paramètres distincts mais complémentaires: complexité, fluidité, correction (Skehan, 2009). Ces paramètres se concurrencent dans la performance du fait de la limitation des ressources attentionnelles disponibles et mobilisées à différents niveaux de traitement. Les résultats obtenus permettent là encore d'obtenir un modèle susceptible d'informer les attentes des enseignants en matière de correction, d'affiner les dispositifs d'évaluation mais aussi d'imaginer des manières d'optimiser l'utilisation des ressources attentionnelles en fonction des tâches.

31 Le choix des priorités va ainsi dépendre des contextes qui façonnent différents profils de locuteur mais aussi de données qui ne sont plus seulement relatives à un statut de locuteur, mais à des motivations de l'apprenant : on peut souligner que la réalité langagière du locuteur ne se confond pas tout à fait avec celle de l'apprenant. Danielle Chini (2010) souligne à juste titre la spécificité de l'enseignement en contexte scolaire : il s'agit de doter l'apprenant d'un moyen de communication pour l'action sociale et de développer des savoir-faire langagiers spécifiques à la langue-culture cible, tout en favorisant la construction individuelle d'un système linguistique qui lui permette de réaliser des performances conformes à l'usage de L2. 


\section{La mise en œuvre sur le terrain de pratiques raisonnées}

Dans le domaine de l'enseignement / apprentissage des langues, de nombreux chercheurs se sont préoccupés de la relation entre les avancées théoriques et la pratique de terrain, déplorant le manque de perméabilité entre les deux sphères ou l'hétérogénéité des savoirs qui leur sont rattachés (Ellis 1997). Si la dimension praxéologique de la DDL est souvent soulignée par les didacticiens, il n'en demeure pas moins que les avancées théoriques sont d'un accès difficile pour les praticiens et qu'à l'inverse, les chercheurs ignorent souvent la réalité du travail de terrain. La posture de l'enseignant engagé dans la recherche (Ellis 2010 : 59-61) permet, au-delà des obstacles et des biais, de résoudre la tension en soumettant des pratiques et des performances à l'investigation. Narcy-Combes et Demaizière (2005) conçoivent la recherche en didactique des langues comme un moyen d'élaborer des pratiques ancrées dans des références théoriques et d'échapper à un fonctionnement qui relèverait plus du conditionnement que de la réflexion (NarcyCombes, $2005: 13)$.

\subsection{La motivation théorique de l'intervention}

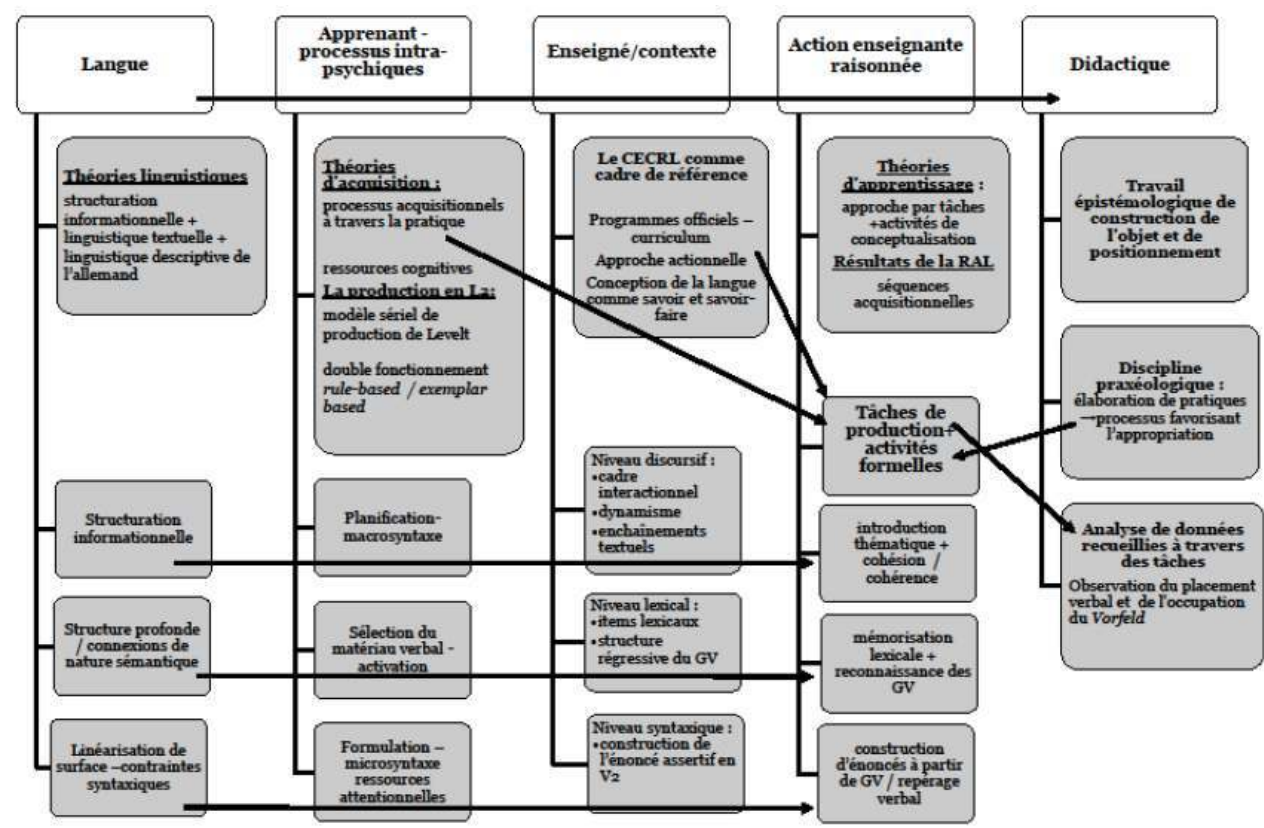

Figure 1 : Articulation théorique d'une recherche en didactique des langues

Le schéma ci-dessus permet de visualiser la construction de notre recherche et de mettre en évidence le système complexe dans lequel les objets propres à chaque domaine de référence interagissent. A chaque domaine sont associés des notions spécifiques dans une lecture verticale, les liens horizontaux matérialisent la conduite de la recherche et l'articulation théorique de la démarche. L'approche par tâches se justifie par rapport à un positionnement théorique qui considère la pratique langagière de L2 comme un moteur de l'appropriation, l'élaboration des activités formelles s'appuie sur la description de L2 dans tous ses aspects et elle vise à influencer les processus de planification, de 
mémorisation, d'activation lexicale et de formulation tels qu'ils sont décrits dans le modèle de Levelt. La problématique est posée à l'intérieur d'un cadre théorique qui intègre des notions issues de chaque domaine de référence et dans une perspective praxéologique, la méthodologie sera liée à une action menée sur le terrain par le biais de tâches associées à des activités formelles. Ces tâches seront destinées à la fois à l'intervention et au recueil des performances qui seront analysées.

\subsection{Les tâches comme construit partagé}

Dans le cadre de notre démarche il nous est apparu particulièrement pertinent d'avoir recours à des tâches dans la mesure où il s'agit d'un « construit » qui possède une validité à la fois sur le plan théorique et sur le plan institutionnel. En ce sens, l'approche par tâches permet dans sa mise en œuvre de faire le lien entre théorie et pratique sans qu'il s'agisse d'une application unidirectionnelle de la recherche sur le terrain. Dans le travail à partir de tâches - que ce soit à des fins scientifiques ou pédagogiques - les intérêts sont partagés puisqu'il s'agit pour le chercheur comme pour l'enseignant de les élaborer de manière à promouvoir l'apprentissage. (Ellis, $2003: 34$ )

Les tâches représentent ainsi pour nous un moyen d'intervention en adéquation avec notre positionnement théorique qui fait de la pratique de L2 une condition essentielle au développement des processus acquisitionnels, elles s'intègrent également dans une approche légitimée par les nouvelles orientations de l'enseignement institutionnel et, dans une perspective acquisitionniste, elles constituent enfin un dispositif pour le recueil de données destinées à être analysées. Nous avons fait le choix de nous appuyer sur la définition que donne Bygate de la tâche pédagogique comme activité structurée, orientée vers un but, mettant en jeu un traitement du langage et ayant une finalité d'apprentissage. (Bygate, 2000). C'est ce dernier point qui nous a paru essentiel afin d'éviter de ne réduire la tâche qu'à son résultat opérationnel (aim) au risque de manquer l'objectif développemental (outcome).

[...] le véritable but de la tâche n'est pas que les apprenants la réalisent avec succès mais qu'ils utilisent la langue de telle manière que cette utilisation favorise l'apprentissage de la langue ${ }^{13}$. (Ellis $2003: 8$ )

\subsection{La posture du chercheur-enseignant}

Ellis $(1997,2010)$ souligne la difficulté de trouver des points de convergence et des passerelles entre les domaines de la théorie et de la pratique. Non seulement les différences de nature entre savoir scientifique et savoir pratique participent de ce clivage et du manque de transfert de connaissances entre recherche et action. Mais en plus, les préoccupations des enseignants et des chercheurs ne se rejoignent pas toujours. Ellis voit dans la recherche effectuée par l'enseignant une possible synthèse. Il préconise ainsi un enseignement qui prendrait appui sur la recherche: en adoptant la posture d'un " chercheur-appliqué » (applied researcher) (Ellis, 2010: 63), l'enseignant peut trouver des solutions à des problèmes concrets liés à sa pratique et dans le même temps développer sa formation personnelle. Cette démarche rejoint le propos de Narcy-Combes sur la responsabilité épistémologique de l'enseignant de « se constituer un savoir et une pratique instruite » (Narcy-Combes, $2005: 85$ ). 


\section{Conclusion : Faire dialoguer les théories, les modèles, les savoirs, les pratiques}

Bien plus qu'une juxtaposition de regards, il s'agit pour nous de rendre justice aux différentes facettes qui interagissent au sein d'un objet complexe (Narcy-Combes 2005). Dans notre contribution nous avons essayé de montrer dans quelle mesure notre démarche de recherche est liée à un positionnement par rapport à la langue et aux choix théoriques que ce dernier conditionne. Des modèles et des notions empruntés aux travaux de chercheurs en RAL sont au cœur de notre réflexion. Elles s'associent à une analyse linguistique qui dépasse la simple description formelle et transforment ou complètent des pratiques éprouvées. C'est dans ce dialogue que se constitue pour nous le savoir enseignant susceptible de faire des activités d'enseignement / apprentissage un « bricolage » éclairé. (Narcy-Combes $2010: 120$ ).

\section{BIBLIOGRAPHIE}

Bange, Pierre (en coll. avec Carol, Rita et Griggs, Peter) (2005), L'Apprentissage d'une langue étrangère: cognition et interaction, Paris: Editions L'Harmattan.

Blumenthal, Peter (1997), Sprachvergleich Deutsch-Französisch, Romanistische Arbeitshefte. Tübingen: Niemeyer.

Bygate, Martin, (2000), « Introduction », in Language Teaching Research. Vol. 4, n³, 185-192.

Chini, Danielle (2010), « Langage et/ou action? La perspective actionnelle favorise-t-elle vraiment l'apprentissage linguistique? ", Le français dans le monde - Recherches et applications. $\mathrm{n}^{\circ}$ 48, 164-175.

Dalmas, Martine (2005) « Dringend nötig: der "Blick über den Satzrand" », in Kirsten Adamzik \& Wolf-Dieter Krause (éd.) Text-Arbeiten. Textsorten im fremd-und muttersprachlichen Unterricht an Schule und Hochschule, Europäische Studien zur Textlinguistik, Tübingen: Gunter Narr Verlag, 97-109.

Diehl, Erika, Christen, Helen, Leuenberger, Sandra, Pelvat, Isabelle \& Studer, Thérèse (2000), Grammatikunterricht - Alles für der Katz? Untersuchungen zum Zweitsprachenerwerb Deutsch, Tübingen : Niemeyer, RGL 220.

Dörnyei, Zoltán (2009), The Psychology of Second Language Acquisition. Oxford; New York: Oxford University Press.

Dürscheid, Christa (2010), Syntax. Grundlagen und Theorien, UTB Sprachwissenschaften, Göttingen: Vandenhoeck \& Ruprecht.

Ellis, Nick C. (2011), «Implicit and Explicit SLA and Their Interface », in Cristina Sanz \& Ronald P. Leow (éd.), Implicit and Explicit Language Learning: Conditions, Processes, and Knowledge in SLA and Bilingualism. Georgetown University Press, 35-47.

Ellis, Rod (1997), SLA Research and Language Teaching, Oxford: Oxford University Press.

Ellis, Rod (2003), Task-based Language Learning and Teaching. Oxford: Oxford University Press, 
Levelt, Willem J.M. (1989), Speaking: From Intention to Articulation. Cambridge: MIT Press.

Meisel, Jürgen, Clahsen Harald \& Pienemann, Manfred (1981), « On determining developmental stages in natural second language acquisition ", Studies in Second Language Acquisition n³ 3 109-135.

Narcy-Combes, Jean-Paul (2005), Didactique des langues et TIC: vers une recherche-action responsable, Paris: Editions Ophrys.

Narcy-Combes, Jean-Paul \& Demaizière, Françoise (2005), « Méthodologie de la recherche didactique : nativisation, tâches et TIC », Alsic. Vol. 8, n¹, 45-64.

Narcy-Combes, Jean-Paul (2010), « Illusion ontologique et pratique réflexive en didactique des langues », Le français dans le monde - Recherches et applications. ${ }^{\circ} 48$, p. 111-122.

Pienemann, Manfred (1999), Language processing and second language development: Processability Theory, Amsterdam: John Benjamins.

Pienemann, Manfred (éd.) (2005), Cross-linguistics aspects of Processability Theory, Amsterdam: John Benjamins.

Poitou, Jacques (2010), « Enquêtes et réflexions sur la topologie de la proposition allemande », Lylia (49), 1-33.

Samson, Gunhild (2004), "L'attaque de l'énoncé assertif en allemand », in Pierre Cotte, Martine Dalmas \& Hélène Włodarczyk (éd.). Énoncer: l'ordre informatif dans les langues. Paris : l'Harmattan, 185-206.

Schanen, François, \& Confais, Jean-Paul (1989), Grammaire de l'allemand: formes et fonctions, Paris: Nathan.

Skehan, Peter \& Foster, Pauline (2001), « Cognition and Tasks », in Peter Robinson (éd.), Cognition and Second Language Instruction, Cambridge: Cambridge University Press, 183-205.

Skehan, Peter (2009), « Modelling Second Language Performance: Integrating Complexity, Accuracy, Fluency, and Lexis », Applied Linguistics, Vol. 30, n 4, 510 -532.

Véronique, Georges Daniel (2005), « Les interrelations entre la recherche sur l'acquisition du français langue étrangère et la didactique du français langue étrangère ", Acquisition et interaction en langue étrangère, (23), 9-41.

Véronique, Daniel (2010), « La recherche sur l'acquisition des langues étrangères: entre le nomologique et l'actionnel », Le français dans le monde - Recherches et applications, $\mathrm{n}^{\circ} 48,76-85$.

von Stutterheim, Christiane et al. (2002), « Différences translinguistiques dans la conceptualisation des événements », Revue française de linguistique appliquée, Vol. VII, n², 99-115. Zifonun, Gisela, Hoffmann, Ludger, Strecker, Bruno \& Ballweg, Joachim (1997), Grammatik der deutschen Sprache, Berlin / New-York : Walter de Gruyter.

\section{NOTES}

1. En référence à Narcy-Combes (2005) et Narcy-Combes \& Demaizière (2005) qui soulignent la nécessité d'un ancrage des pratiques dans des références théoriques et l'importance de la réflexion épistémologique dans les choix et l'articulation théoriques de la recherche en didactique.

2. Terme inapproprié pour le linguiste germaniste et l'enseignant de langue qui s'appuient sur une description de l'allemand qui s'est depuis Jean Fourquet affranchie du modèle donné par 
l'ordre canonique SVO du français. Nous conserverons néanmoins ce terme d' "inversion » pour désigner - comme il est d'usage dans la littérature relative à l'acquisition de l'ordre des mots l'opération consistant à positionner le sujet dans le Mittelfeld après la forme fléchie du verbe.

3. Schanen et Confais parlent d'avant-première et d'après-dernière position pour désigner les champs respectivement en amont et en aval des limites de l'énoncé (Schanen \& Confais, 1989 : 577-579).

4. Termes employés à dessein et qui recouvrent des notions dont les contours varient selon les théories ou les écoles de référence et que nous ne développerons pas ici. (given/ new - topique/ commentaire - arrière-plan/focus - théme/rhème)

5. «Es geht um die Absicht des Sprechers, mit grammatischen Mitteln Schwerpunkte in seiner Äußerung zu setzen, verschiedene Wichtigkeitsgrade der Wörter zu unterscheiden und so die Mitteilungswerte im Satz abzustufen. »

6. L'accent contrastif marquant la prosodie de la phrase est indiqué par le signe ${ }^{\circ}$.

7. Conformément au titre de son article « der Blick über den Satzrand " [aller jeter un œil au-delà des limites de la phrase].

8. « (...)während diese [die Besetzung der ersten Stelle im Satz] in den Subjekt-prominenten Sprachen ausschließlich nach Kriterien der morphologisch-kongruentiellen Syntax (SubjektKongruenz mit dem Verb) geregelt ist, erfolgt im Deutschen die Besetzung der ersten Stelle innerhalb des Satzes unter textsyntaktischen Gesichtspunkten: die alte Information (Topic) bildet den Anknüpfungspunkt zum Prätext und steht satzinitial. »

9. «Als Anfang einer Thema-Rhema-Einheit im Aussage-Modus ist das Vorfeld in besonderer Weise geeignet, den thematischen Anschluss an das Vorangehende herzustellen, das heißt, es ist der bevorzugte Platz für Komponente, mit denen ein Thema fortgeführt oder entwickelt wird. (... ).Thematizität ist nicht an bestimmte syntaktische Einheiten gebunden. »

10. On peut se reporter ici au schéma de Narcy-Combes (2005: 41) illustrant la relation de transduction entre la langue système, la langue outil et la réalisation physique que représente le discours.

11. Il faut ici souligner que ce savoir est un savoir de spécialiste, il n'est pas destiné aux apprenants mais constitue une base que l'enseignant se construit afin d'être capable de construire par la suite de façon réfléchie avec les apprenants. En ce sens il relève de la responsabilité épistémologique que nous évoquons plus loin en référence à Narcy-Combes (2005). 12. "The task of acquiring a language includes the acquisition of the procedural skills needed for the processing of the language. It follows from this that the sequence in which the target language (TL) unfolds in the learner is determined by the sequence in which processing routines develop which are needed to handle the TL's components. »

13. "[...] the real purpose of the task is not that learners should arrive at successful outcome but that they should use language in ways that will promote language learning."

\section{RÉSUMÉS}

Comment appréhender la langue comme objet d'apprentissage dans le cadre institutionnel tout en rendant justice aux multiples facettes qui composent cet objet complexe, aux enjeux communicatifs et linguistiques tels qu'ils sont posés dans les prescriptions officielles et aux objectifs de la recherche didactique en tant que discipline praxéologique ? Le cadre théorique qui 
sous-tend notre recherche s'est construit à partir de ces questions et convoque ainsi autour de l'objet-langue et de son appropriation un faisceau de théories issues de domaines connexes. Tout en présentant l'articulation théorique de notre travail, il nous importe de mettre en évidence ce qu'une approche plurielle de la langue et une prise en compte des travaux issus de la Recherche en Acquisition des Langues (RAL) sont susceptibles d'apporter aussi bien au didacticien qu'à l'enseignant de langue.

In the field of didactics, research aims to have a praxeological dimension, i. e. that the findings should have empirical implications and should contribute to promote acquisition in terms of an appropriate use of the L2. How to define "language" as a scientifically constructed object and as an object that has to be taught in order to be used? In the theoretical framework of our research, we draw on several theories from different related disciplines such as Linguistics and SLA and assume that a multiple perspective on language (linguistic, cognitive and functionalist) and an integration of models and methods developed in SLA and psycholinguistics are crucial for both the researcher and the language teacher.

\section{INDEX}

Keywords : didactics, epistemology, syntactic acquisitions, information structure, processing, tasks, German L2.

Mots-clés : didactique, épistémologie, acquisitions syntaxiques, structure informationnelle, processus cognitifs, tâches, allemand L2.

\section{AUTEUR}

\section{CATHERINE FELCE}

Université Sorbonne Nouvelle - Paris 3 - DILTEC

c.felce@orange.fr 